\title{
HEATING MECHANISMS FOR INTERMITTENT LOOPS IN ACTIVE REGION CORES FROM AIA/SDO EUV OBSERVATIONS
}

\author{
A. C. Cadavid ${ }^{1}$, J. K. Lawrence ${ }^{1}$, D. J. Christian ${ }^{1}$, D. B. Jess ${ }^{2}$, and G. Nigro ${ }^{3}$ \\ ${ }^{1}$ Department of Physics and Astronomy, California State University Northridge, Northridge, CA 91330, USA \\ ${ }^{2}$ Astrophysics Research Centre, School of Mathematics and Physics, Queen's University Belfast, Belfast BT7 1NN, UK \\ ${ }^{3}$ Universita della Calabria, Dipartimento di Fisica and Centro Nazionale Interuniversitario Struttura della Materia, \\ Unita di Cosenza, I-87030 Arcavacata di Rende, Italy \\ Received 2014 April 29; accepted 2014 September 5; published 2014 October 13
}

\begin{abstract}
We investigate intensity variations and energy deposition in five coronal loops in active region cores. These were selected for their strong variability in the AIA/SDO $94 \AA$ intensity channel. We isolate the hot Fe XVIII and Fe XXI components of the $94 \AA$ and $131 \AA$ by modeling and subtracting the "warm" contributions to the emission. HMI/SDO data allow us to focus on "inter-moss" regions in the loops. The detailed evolution of the inter-moss intensity time series reveals loops that are impulsively heated in a mode compatible with a nanoflare storm, with a spike in the hot 131 A signals leading and the other five EUV emission channels following in progressive cooling order. A sharp increase in electron temperature tends to follow closely after the hot $131 \AA$ signal confirming the impulsive nature of the process. A cooler process of growing emission measure follows more slowly. The Fourier power spectra of the hot $131 \AA$ A signals, when averaged over the five loops, present three scaling regimes with break frequencies near $0.1 \mathrm{~min}^{-1}$ and $0.7 \mathrm{~min}^{-1}$. The low frequency regime corresponds to $1 / f$ noise; the intermediate indicates a persistent scaling process and the high frequencies show white noise. Very similar results are found for the energy dissipation in a 2D "hybrid" shell model of loop magneto-turbulence, based on reduced magnetohydrodynamics, that is compatible with nanoflare statistics. We suggest that such turbulent dissipation is the energy source for our loops.
\end{abstract}

Key word: Sun: corona

Online-only material: color figures

\section{INTRODUCTION}

One approach to understanding coronal heating has been to study the thermal behavior of coronal loop structures found above and near solar active regions (ARs). Several studies indicate that "warm loops" on the periphery of AR cores, with temperatures around $1 \mathrm{MK}$, have narrow temperature distributions (Aschwanden \& Nightingale 2005; Bradshaw 2008; Tripathi et al. 2009) and that they evolve in time from hotter to cooler temperatures (Ugarte-Urra et al. 2009; Mulu-Moore et al. 2011). In contrast, structures in the AR core, including the loop apexes (Warren et al. 2011) and inter-moss areas between moss patches of opposite polarity (Warren et al. 2012), have been found to remain steady over periods of hours. Earlier studies of magnetic moss regions (Antiochos et al. 2003) have found steady heating, but recent observations with the High Resolution Coronal Imager (Hi-C) indicate high variability in moss regions at the footpoints of hot coronal loops (Testa et al. 2013). In other work, Ugarte-Urra et al. (2009) and Viall \& Klimchuk (2011, 2012) have found that in the AR core the loop structures are not steady and that they evolve from hotter to cooler temperatures.

Much research has focused on relating observations to heating via impulsive bursts or "nanoflares" (Klimchuk 2006, and references therein). The bursts might be the result of reconnection events among braided fields as in the original nanoflare scenario of Parker (1988), or possibly due to the dissipation of magnetohydrodynamic (MHD) turbulence inside the loop structures (Nigro et al. 2004; Reale et al. 2005; Veltri et al. 2005; van Ballegooijen et al. 2011; Asgari-Targhi \& van Ballegooijen 2012; Asgari-Targhi et al. 2013). Within the nanoflare model different outcomes follow from different heating rates of the subresolution loop strands. In a high-frequency process the time between heating impulses is shorter than the cooling times of the strands, thus smoothing the changes over a period of time. This process can be referred to as "steady heating." In the case of low-frequency heating the time between bursts is longer than the cooling time of the structure, and the strands evolve individually to cooler temperatures. Such a process is labeled "nanoflare heating" (Cargill \& Klimchuk 1997; Tripathi et al. 2011; Winebarger et al. 2011).

Within this class of processes is a scenario in which the loop consists of a small number of strands that are all heated at about the same time. The observed loop properties follow the time evolution of the strands in this "short nanoflare storm" (Klimchuk 2009). Alternatively the loop can be made of many strands all heated independently at random times. In this "long nanoflare storm" (Klimchuk 2009) the loop properties can appear steady due to averaging of the contributions over many strands. Actually, the problem is more subtle in that both the low and high frequency processes can dominate at different stages during the long term evolution of an AR (Ugarte-Urra \& Warren 2012). Independent of the details, all nanoflare heating scenarios predict the presence of very hot plasma in the coronal loops. Although Reale \& Orlando (2008) have shown the difficulty of detecting the hot plasma outside of flaring regions, a variety of studies using data from Hinode, RHESSI, and the Solar Dynamics Observatory (SDO) have applied various techniques to discover very high temperatures in loop like structures in such nonflaring active regions (e.g., Patsourakos \& Klimchuk 2009; Reale et al. 2009, 2011; Testa et al. 2011; Testa \& Reale 2012; Petralia et al. 2014).

Ugarte-Urra \& Warren (2014, henceforth UW14) have approached the problem of the energy deposition in ARs by investigating the statistical properties of heating events defined by AIA 
intensity signals in the $94 \AA$ channel, in which the $\sim 10^{6.85} \mathrm{MK}$ hot component from the Fe XVIII ions has been isolated. They find a minimum frequency of 2-3 heating events per hour. Using the "Enthalpy-based Thermal Evolution of Loops" (EBTEL) zero dimensional hydrodynamic coronal model (Klimchuk et al. 2008), they investigate cases with heating events of different frequencies. These were assumed to be square pulses of random amplitudes obtained from a power law distribution, with a constant event duration of $\sim 200 \mathrm{~s}$, and with times randomly chosen from a normal distribution. The results indicated that the actual frequency of the heating events can be higher than those "observed" since these synthetic intensities integrate over the contributions of a multiplicity of events.

In this paper we approach the problem of energy release into the corona by investigating the dynamics of five "hot" loops in AR cores that were initially selected for the high temporal variability of their intensities in the $94 \AA$ channel. To investigate the properties of energy deposition and the hot loop evolution we use data from the AIA/SDO instrument (Lemen et al. 2012) and consider the six Extreme Ultraviolet (EUV) channels: 131, $94,335,211,193$, and $171 \AA$. We also use contemporaneous data from the HMI/SDO instrument to define what we call the "inter-moss" region in the loop structures. From spatial averages along these loop inter-moss segments we obtained the intensity time series that we analyzed for this project.

We find that the nonsteady loops of the kind under study are impulsively heated in a mode compatible with a nanoflare storm. Abrupt loop intensity brightenings typically begin with a sharp impulse in the hot $131 \AA$ intensity followed in sequence by progressively broader, smoother, and later increases in the hot $94 \AA$ intensity and then the $335 \AA$ intensity. When there is enough time between heat impulses, the cooler signals in the $211 \AA, 193 \AA$, and $171 \AA$ are also observed to increase in that progressive order from hotter to cooler. A sharp increase in loop electron temperature tends to follow quickly after the hot $131 \AA$ signal, confirming the impulsive nature of the process. Since the hot $131 \AA$ intensity is the least affected by processes within the loop, such as cooling and plasma flows, it is our best choice as a proxy for energy input. The power spectra of the hot $131 \AA$ intensity fluctuations and the energy dissipation in an MHD turbulence model (Nigro et al. 2004; Reale et al. 2005) both show the characteristics of a $1 / f$ process for the lower frequencies, "strong persistence" for the intermediate regime, and white noise at higher frequencies. This reaffirms the use of the hot $131 \AA$ intensity to investigate the energy release process in the corona, and it suggests that the energy source for the observed intensity increases is dissipation from MHD turbulence. Based on the location of the brightenings we conjecture that the energy release is occurring at the loop tops.

In Section 2 we introduce the observations and techniques used to prepare the data for later analysis, including the identification of the hot signals. In Section 3 we describe how the loops are identified, including our background subtraction method, and the definition of the intensity time series. Section 4 presents the analysis of the patterns of loop intensity fluctuations and the comparison to modeled results. Section 5 details our loop heating scenario, and Section 6 presents the scaling properties of the loop energy input. Lastly, in Section 7 we summarize our findings and further discuss the implication of the results.

\section{DATA}

We use data in the six Extreme Ultraviolet (EUV) channels $(131,94,335,211,193,171 \AA)$ from the Atmospheric Imag-
Table 1

Temporal and Spatial Properties for Loops A-E

\begin{tabular}{lcccc}
\hline \hline Loop & Date & Starting Time & $\begin{array}{c}\text { Length } \\
(\mathrm{Mm})\end{array}$ & $\begin{array}{c}\text { Inter-Moss Limits } \\
(\mathrm{Mm})\end{array}$ \\
\hline A & 2011 Jul 15 & UT 11:32:00 & 30.5 & $10.9-23.9$ \\
B & 2011 Jul 14 & UT 12:02:00 & 32.2 & $13.0-21.8$ \\
C & 2014 Jul 13 & UT 12:02:00 & 24.8 & $7.8-15.7$ \\
D & 2014 Jul 13 & UT 12:02:00 & 33.5 & $16.5-26.1$ \\
E & 2014 Jul 13 & UT 12:02:00 & 23.9 & $10.9-19.6$ \\
\hline
\end{tabular}

ing Assembly (AIA; Lemen et al. 2012) on board the SDO (Pesnell et al. 2012). This is in virtually continuous operation and covers the full solar disk with a $0^{\prime \prime} 6(\sim 0.44 \mathrm{Mm})$ pixel scale (spatial resolution of $1^{\prime \prime} .2$ or $\sim 0.9 \mathrm{Mm}$ ) and a cadence of $12 \mathrm{~s}$. The Helioseismic and Magnetic Imager (HMI) instrument on board $S D O$ provides contemporaneous, full-disk, line-of-sight, photospheric magnetic data at a cadence of $45 \mathrm{~s}$. These locate sites of underlying magnetic moss so that their fluctuations can be excluded from the observations of loop intensity changes.

The class of events under study was discovered by means of animations of series of AIA/SDO observations of NOAA AR 11250 during its first disk passage. The observations were made on 2011 July 13, 14, and 15. On July 14, AR 11250 crossed the solar meridian at S27. The Heliophysics Events Knowledgebase (http://lmsal.com/hek/hek_isolsearch.html) reports no observations of flares in this AR during the time intervals we are studying. Likewise, no GOES flares are reported during these times. The data did reveal a number of transient $94 \AA$ brightenings along short ( $\sim 20-30 \mathrm{Mm})$ magnetic loops in the AR core that connect areas of opposite polarity moss. Here we present the detailed analysis of the times series of length 270 minute for five loops labeled A-E. Table 1 gives their dates, starting times, length of the loops, and the boundaries of the inter-moss regions. Figure 1 shows images in the 94 and $131 \AA$ signals for Loop D at $\sim 50$ minutes after the starting time of the series at UT 12:02.

To investigate energy deposition in the AR we isolate the "hot" components of this channel and also of the $131 \AA$ A channel. The AIA $94 \AA$ response function has two maxima: a "hot" component at $T \approx 10^{6.85} \mathrm{~K}$, due to the Fe XVIII ion, and a "warm" component at $T \approx 10^{6.2} \mathrm{~K}$ (Boerner et al. 2014). Reale et al. (2011) extract the hot $94 \AA$ signal by subtracting a fraction of the $171 \AA$ signal that is used to model the "warm" component (which these authors label "cool"). Warren et al. (2012) and UW14 model the warm component of the $94 \AA$ emission in terms of a combination of the $171 \AA$ and the $193 \AA$ emission by introducing a quartic polynomial in the quantity $x=f I_{171}+(I-f) I_{193}$. Our variant of the approach to defining the hot component of the signal is to take it to be $I_{94 \mathrm{~h}}=I_{94}-a x$. To find the factor $f$ and the coefficient $a$ we use trial and error to minimize the absolute value $\left|I_{94 \mathrm{~h}}\right|$ averaged over regions away from the AR core and over all times in the 270 minute data block. For example, for the observations in 2011 July 15 we obtain $f=0.19$ and $a=0.0051$. We note that Del Zanna (2013) found an estimate of the Fe XVIII contribution to the $94 \AA$ signal by modeling the "warm" component as a linear combination of the 171 and the $211 \AA$ A bands. The $131 \AA$ A response function also has two maxima: a "hot" component at $T \approx 10^{7.05} \mathrm{~K}$ corresponding to emission by Fe XXI ions, and a "warm" component centered at $T \approx 10^{5.75} \mathrm{~K}$. The relatively cool temperature of the "warm" part of the $131 \AA$ response function overlaps only the $171 \AA$ response function. In 

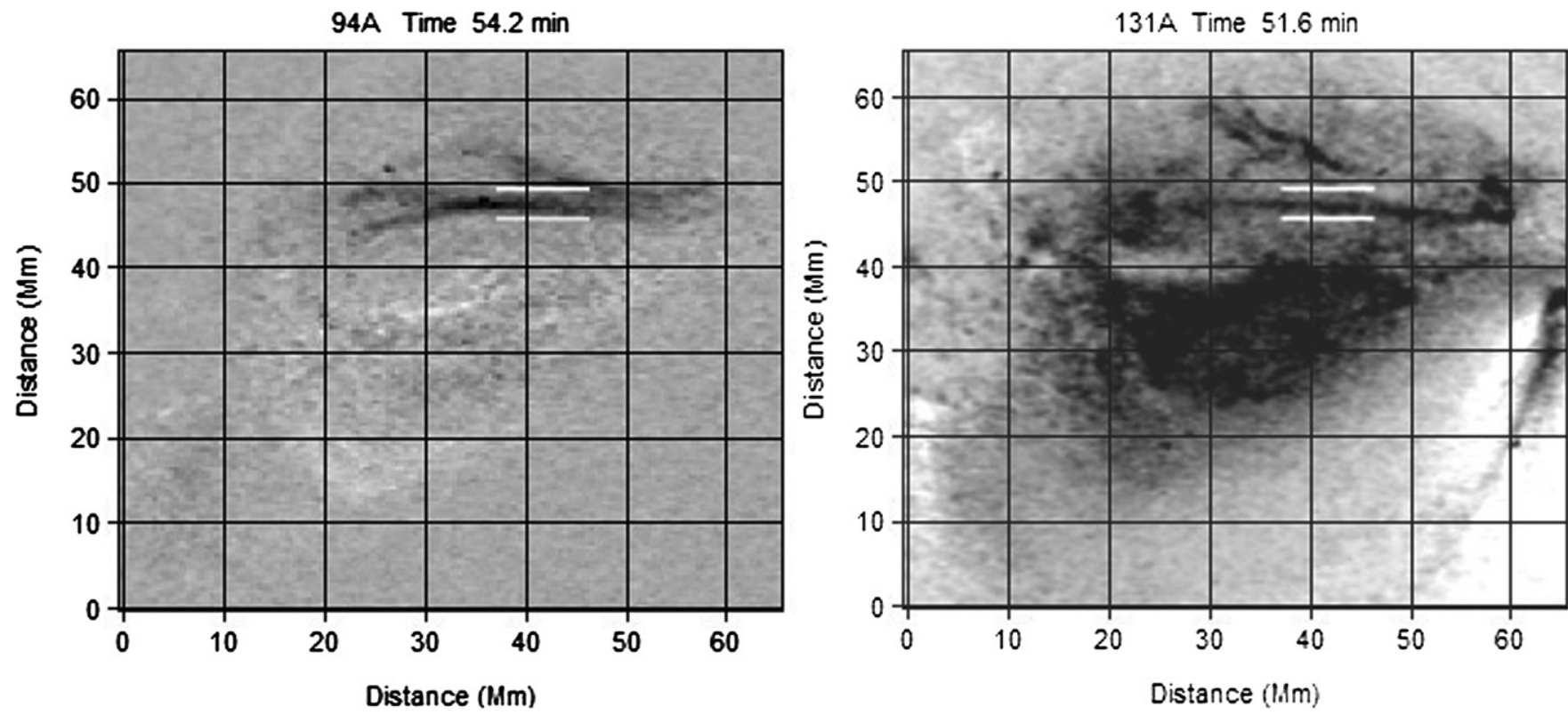

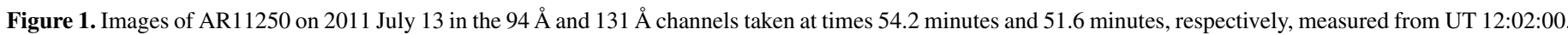
The grayscale is reversed. The white lines indicate the Loop D inter-moss region studied.
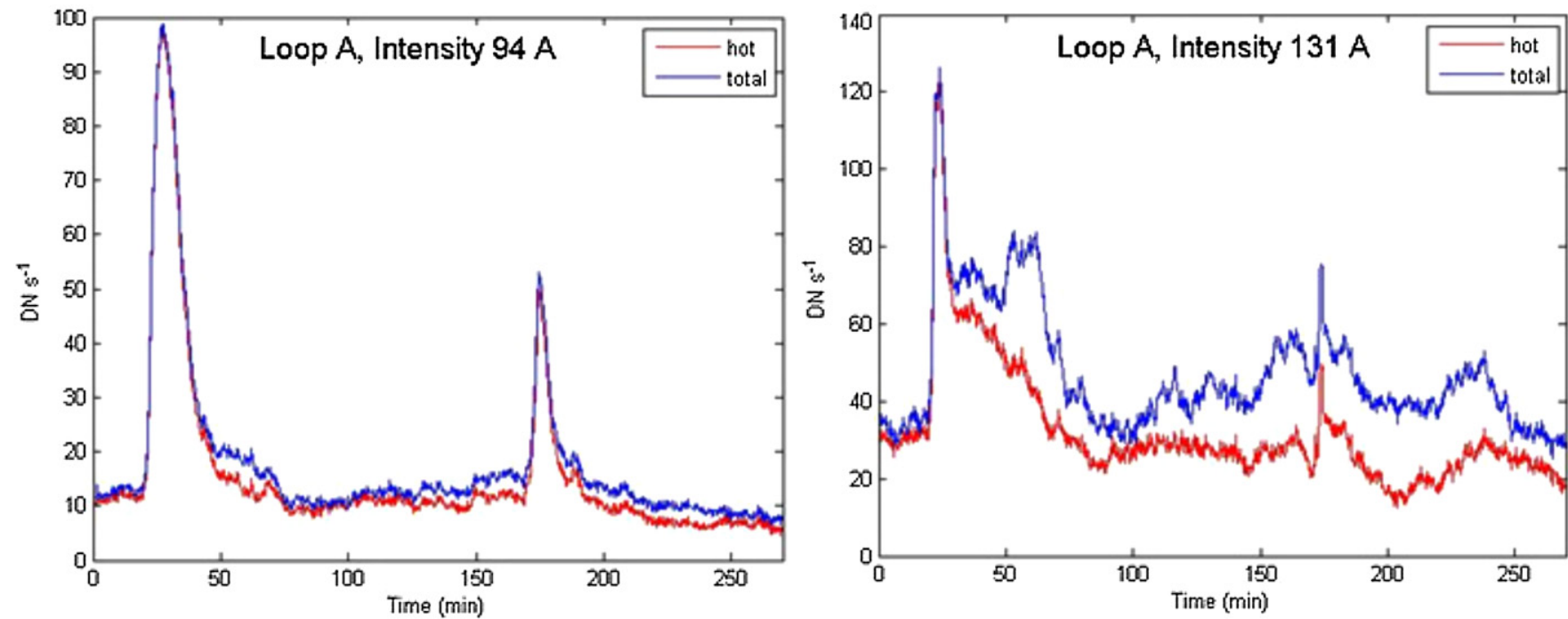

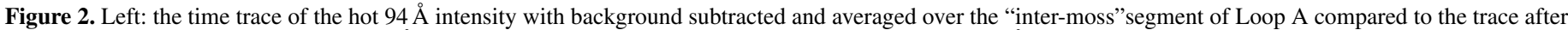
subtraction of the warm portion of the $94 \AA$ response function. Right: the equivalent traces for the hot $131 \AA$ intensity.

(A color version of this figure is available in the online journal.)

this case we model the hot emission as $I_{131 \mathrm{~h}}=I_{131}-a I_{171}$, and we implement a minimization procedure similar to that for the $94 \AA$ case. We obtain a value of $a=0.044$ for the 2011 July 15 data. The values of the scaling factors have variations depending on the quiet region away from the AR core chosen to perform the analysis. For the $131 \AA$ case one of the lowest scaling factors encountered was $a=0.032$. Within these limits we find that the choice of factor does not significantly affect results presented later in the paper. The $a=0.044$ choice is near the ratio of the filter response functions which gives 0.047 . In the case of the $94 \AA$ channel the ratio of the filter response functions leads to $a=0.0071$. These models for the warm components, especially the $131 \AA$ case, are by necessity coarse approximations. However they appear quite effective in allowing us to separate the approximate contributions of the hot Fe XVIII
(93.93 $\AA$ ) and the hot Fe XXI (128.75 $\AA$ ) which dominate the emission in the signals during the impulsive phases of the loop brightenings. We have found no indication of a hot component in the $193 \AA$ bandpass representing temperature $\approx 2 \times 10^{7} \mathrm{~K}$. Figure 2 displays time traces of the $131 \AA$ and $94 \AA$ "total" and "hot" signals for the background-subtracted and spatially averaged inter-moss segment of Loop A (which will be defined in the next section and which we will use as a prototype). In the $94 \AA$ intensity time trace the hot component dominates throughout this signal. In contrast, in the $131 \AA$ intensity time trace the hot signal dominates the first brightening but the warm contribution is large at other times and dominates the wide intensity peak centered at $\sim 56$ minutes.

The loops are defined by the brightenings in the $94 \AA$ images in a nonflaring region. To estimate the energy content and 

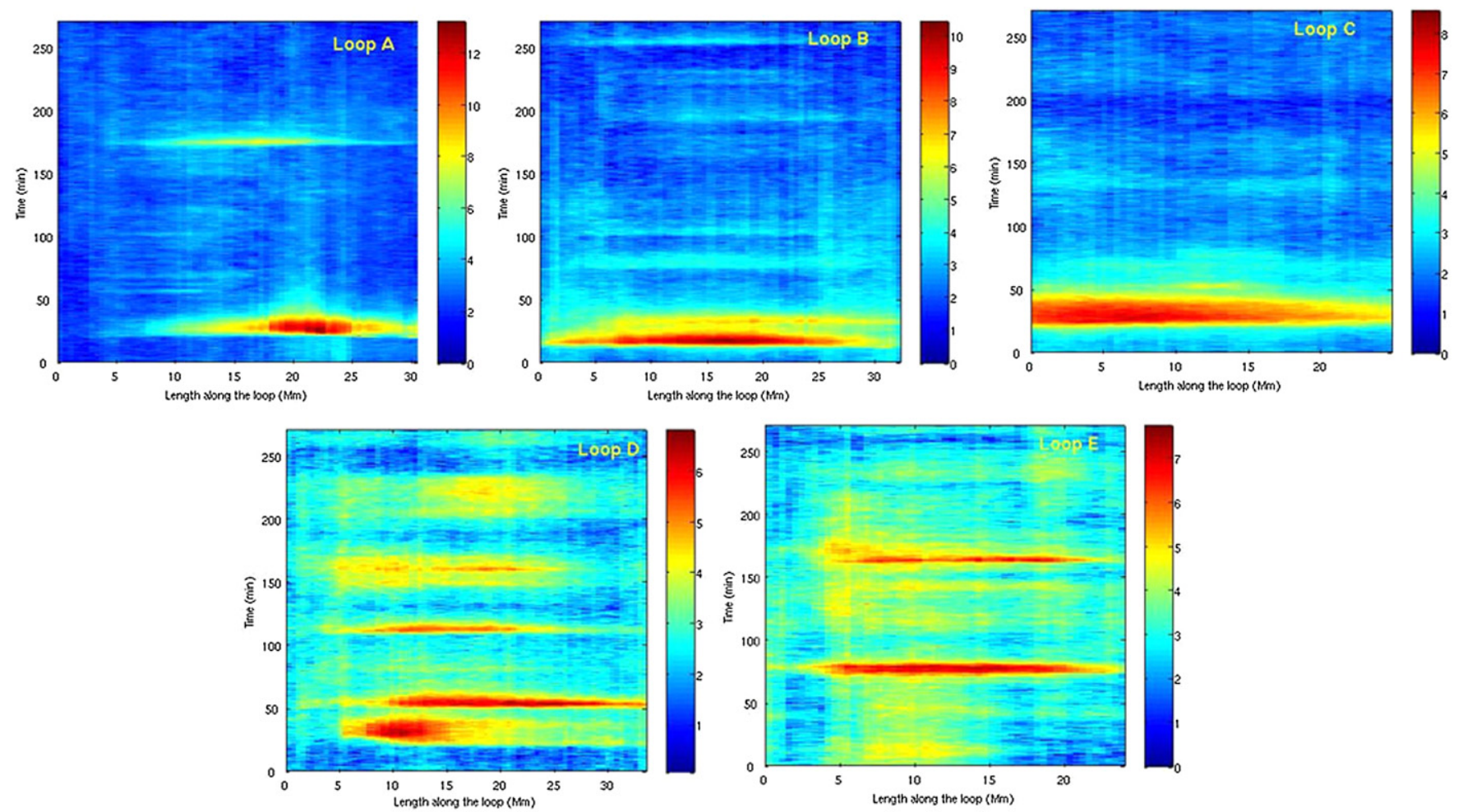

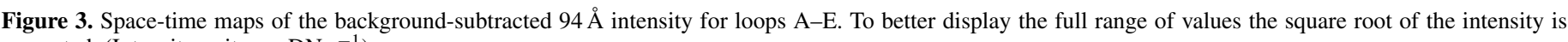
presented. (Intensity units are $\mathrm{DN} \mathrm{s}^{-1}$ ).

(A color version of this figure is available in the online journal.)

compare it to the flare luminosity scale we have applied the following procedure. For a given time series of images we selected a $512 \times 512$ pixel region which included the AR under study and summed the intensity over all pixels. From this "space integrated" intensity time series we then subtracted a background level so that each value is the space integral of the emission in the image. We then identified the maximum value in the time series as a measure of the energy in the emission event. To calibrate the energy measure we applied the procedure to two time series which included flares. The first event was the GOES C1.2 flare on 2011 March 3, UT 19:24-19:44, which was thoroughly studied by Petkaki et al. (2012) and who found that the $94 \AA$ emission band was dominated by the Fe XVIII line and the $131 \AA$ band by the Fe XxI line, which correspond to the hot contributions. Therefore, to avoid overprocessing we have used the original AIA observations. The peak integrated intensity had a value of $1,824,180 \mathrm{DN} \mathrm{s}^{-1}$ in the $94 \AA$ channel and 3,070,000 in the $131 \AA$ channel. For comparison we also considered the GOES B3.3 flare on 2011 July 13, UT 00:47-00:54, and the corresponding peak integrated intensities were 390,000 (94 ̊̊) and $1,260,000$ (131 $\AA$ ). The procedure was then applied to AR 11250 and we found the following ratios between the peak integrated intensities of the flares and those of the brightenings for loops A and B. For the $94 \AA$ A channel: C1.2/B3.3 = 4.7, C1.2/ Loop $\mathrm{A}=11.6, \mathrm{C} 1.2 /$ Loop $\mathrm{B}=19.5$. For the $131 \AA$ channel: C1.2/B3.3 = 2.4, C1.2/Loop A = 21.1, C1.2/Loop B =39.0. In summary, compared to a $\mathrm{C} 1.2$ flare the larger brightenings in our loops have an intensity that is down by a factor between 10 and 20 for the $94 \AA$ channel, and between 20 and 40 for the $131 \AA$ A channel. The brightenings are down from the B3.3 flare intensity by factors between 2.5 and 4 for the $94 \AA$ case, and between 9 and 16 for the $131 \AA$ case.

\section{LOOP IDENTIFICATION AND DEFINITION OF THE INTER-MOSS REGION}

We define the "spine" of a loop by first identifying the $94 \AA$ image in the time series with maximum loop intensity and then averaging over the 11 images centered on this image in time. Then for each pixel coordinate in the EW direction we find the pixel NS coordinate with maximum average intensity. These EW and NS coordinates of the spines identify loops that are projected, both in the line of sight and in the NS directions, through time. For each image and at each EW pixel of the spine we average over \pm 4 pixels (total width of $3.9 \mathrm{Mm}$ ) from the spine in the NS direction. Thus, at each time we are averaging over an area on the Sun that will (ideally) enclose the whole loop feature, or at least the loop core, through time in the segment of interest. It can accommodate NS loop motion up to $2 \mathrm{Mm}$ in either direction. The averages also will contain significant nonvarying background. The same spine coordinates are used to calculate the loop intensities in the remaining five EUV time series leading to six space-time maps for each loop. Figure 3 maps the loop spine intensities showing the evolution of the five loops in the $94 \AA$ A channel. The "brightenings" characterized by the rapid increase in intensity will be thoroughly studied in the following sections.

In order to reveal the loop intensity changes, we must separate the variable loop signals from the steady background emission. It is well-known that the method used for background subtraction can lead to a noticeable effect on results (e.g., Del Zanna \& Mason 2003; Terzo \& Reale 2010; Aschwanden \& Boerner 2011). An established technique appropriate for steady loops was developed in Klimchuk (2000) in which the background is identified by interpolating across the axis of the loop. In another 

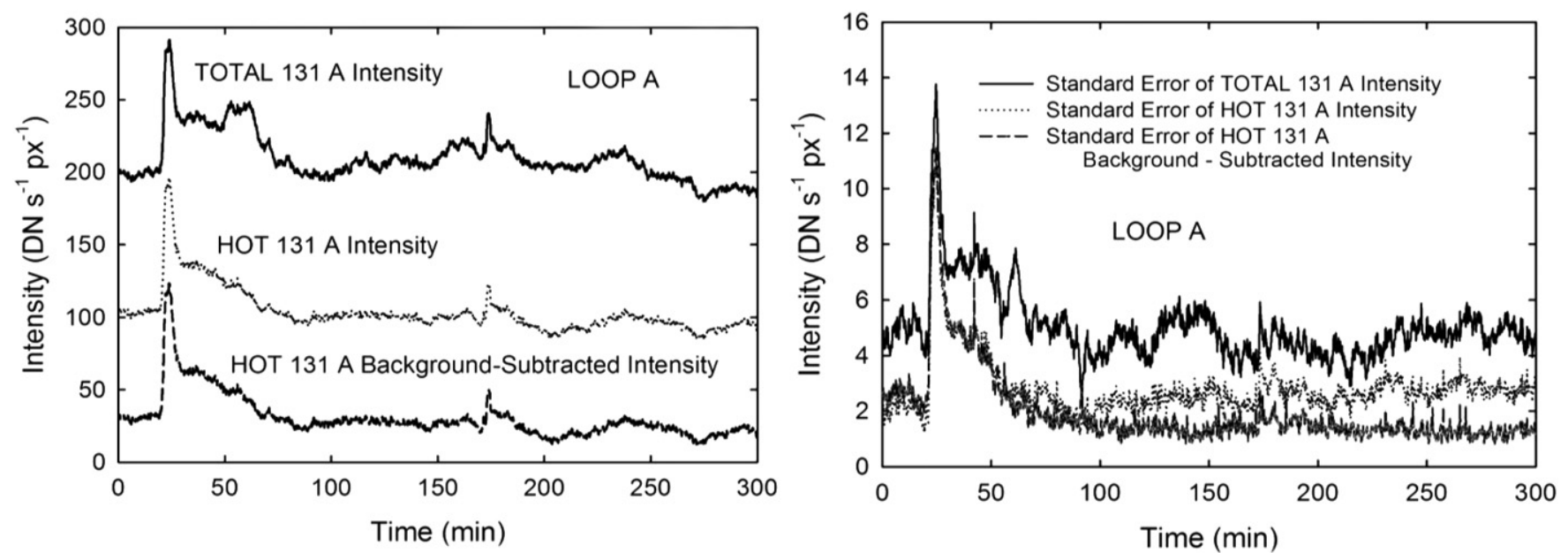

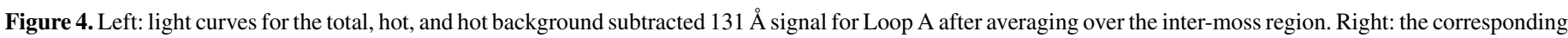
standard errors.

approach Aschwanden \& Boerner (2011) and Aschwanden et al. (2013) perform a fit across each loop point which includes a Gaussian profile and a cospatial linear background. These approaches are based on attempting a spatial isolation of the loop emission from that of underlying plasma.

Our objective here is to investigate the temporal behavior of a particular class of loops. The hot intensities display a variably emitting component superimposed upon a background of steady emission. Thus, we need to concentrate on the variable component and remove the effect of the constant background emission, which may partly reside within the loop itself. So we try a separation based on variability in time rather than in space. For each pixel position along the loop spine, we find the minimum intensity value there through time, and then subtract that minimum value from all values at that pixel location. This procedure is designed to avoid any negative backgroundsubtracted intensity values. However, partly because of noise, the minimum values along a given loop spine come at different times. Thus the intensity averaged over a finite loop segment will show a significantly positive minimum value. See Figure 5.

The subtraction procedure is applied to the data in the six EUV channels. In the study of the dynamics of a small flare, Petkaki et al. (2012) perform a similar temporal background subtraction selecting the value of the lowest total emission in the $94 \AA$ and $131 \AA$ channels. Figure 3 maps the background-subtracted $94 \AA$ intensity along the five loops through time. Loops A and B are characterized by one main "concentrated" brightening with very small subsequent intensity peaks. Loop C exhibits a brightening "interval" with more structure. Loops D and E show multiple brightenings of varying intensity.

Inspection of Figure 3 shows that the major increases in the $94 \AA$ intensity occur around the apex of the loop. In order to identify this segment in the loops we have calculated space time maps of the contemporaneous photospheric line-of-sight (LOS) magnetic field observed with the HMI instrument. The strong positive and negative LOS fields corresponding to the moss regions in the photosphere are localized at distances $<5 \mathrm{Mm}$ and $>25 \mathrm{Mm}$ along the projected Loop $\mathrm{A}$ as seen in Figure 3 (top left). To further refine the limits we have averaged the magnetic field in time. We then define the inter-moss region in the photosphere by the boundaries where the average value of the LOS photospheric field is small. This corresponds to $\sim 11 \mathrm{Mm}$ and $\sim 24 \mathrm{Mm}$ along Loop $\mathrm{A}$ as seen in Figure 3. There are variations among loops. For example for loops B and
D the average photospheric LOS magnetic field is essentially negligible in the photospheric inter-moss region, while for loop E there is no plateau between the two polarities so the boundaries of the inter-moss region are estimated purely from the spacetime intensity maps. Observations that are superimposed on moss show different properties than those we are presenting in this paper. Although they do not reflect the chromospheric or coronal fields, we use the boundaries identified for the photospheric magnetic field to define what we will refer to as the "inter-moss" region in the loops. This is an approximation since we are dealing with the loop projection but it suffices to determine a loop segment away from the moss and near the apex region.

\section{LOOP INTENSITY FLUCTUATIONS}

In order to follow the time evolution of the loops with individual light curves, we have averaged the intensities along the inter-moss segments listed in Table 1. In Figure 4 (left) we summarize and illustrate the effects of the process of selecting the hot component and then subtracting the steady emission background. All three traces are averaged over the inter-moss region for the Loop A, $131 \AA$ signal. In addition to the effects already noted in Figure 2, the intensity for the hot component is about one half of that of the total signal. The background subtraction reduces the intensity level by an additional factor of four. Figure 4 (right) presents the standard error resulting from averaging over the inter-moss region for the three light curves. For the peak of the first brightening the error has essentially the same value in the three cases. For the hot background subtracted case it corresponds to about $10 \%$ of the signal. For the second peak near 170 minutes the error is about $5 \%$ and for the steady segments it can be as large as $8 \%$. Figure 5 presents the resulting time series for the hot components of the $131 \AA$ and $94 \AA$ intensity light curves for the five loops. In all cases the major brightenings are characterized by a peak in the hot $131 \AA$ signal closely followed by a peak in the $94 \AA$ series. The time differences between the two maxima range between 0.8 minutes and 3.2 minutes with an average of 1.5 minutes. The intensities are in the range 40-100 DN s ${ }^{-1}$ pixel $^{-1}$.

To investigate details of the heating mechanism we have followed the evolution of the intensity averages in all of the EUV channels. Figure 6 presents three examples of brightenings in which the energy initially released in the hot $131 \AA$ is followed 

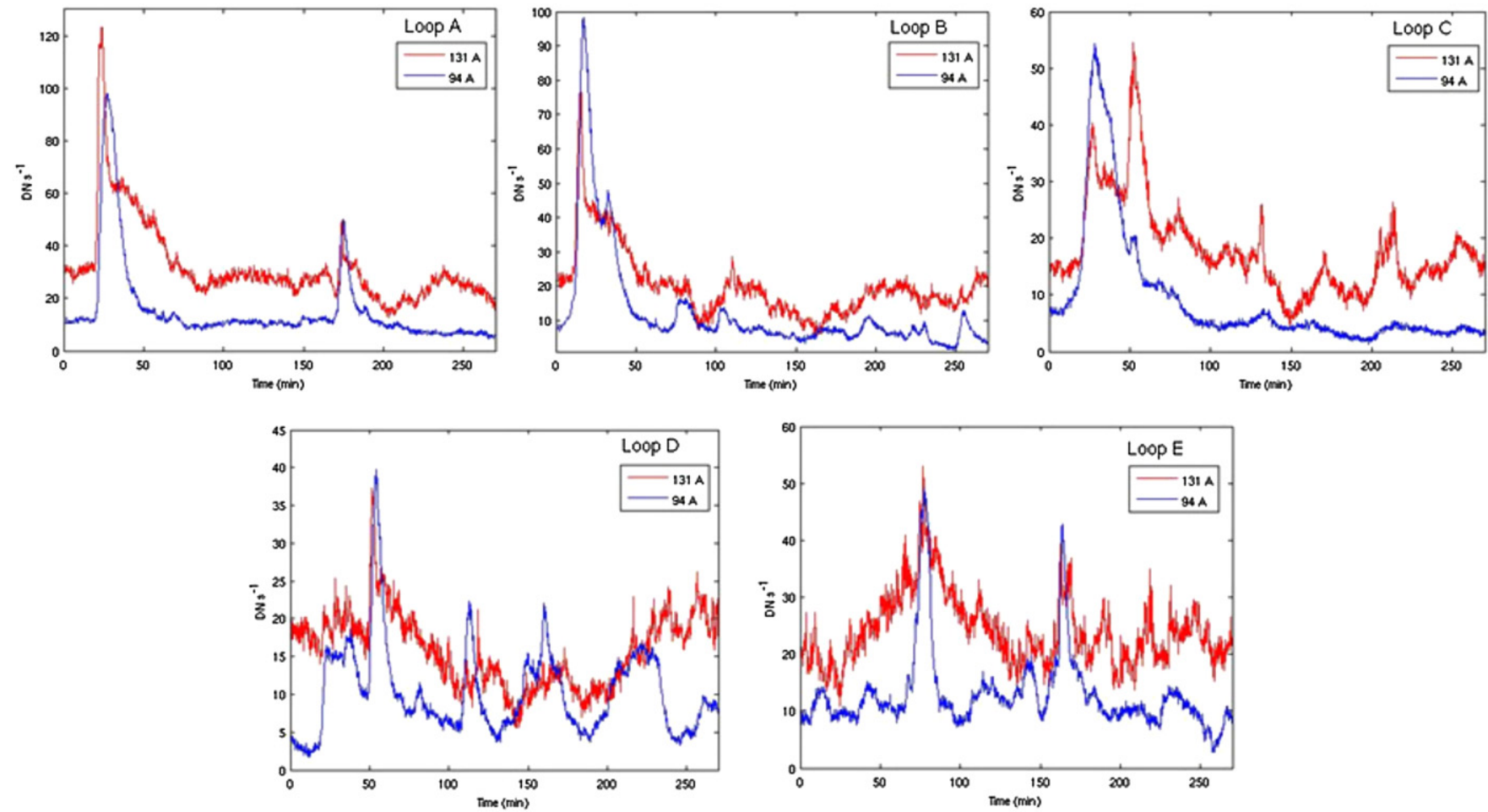

Figure 5. Intensity time lines of the hot $131 \AA \AA$ and $94 \AA$ signals averaged over the inter-moss parts of each of the five loops. The peaks in the $94 \AA$ channel correspond to the brightenings in the space-time maps in Figure 3.

(A color version of this figure is available in the online journal.)
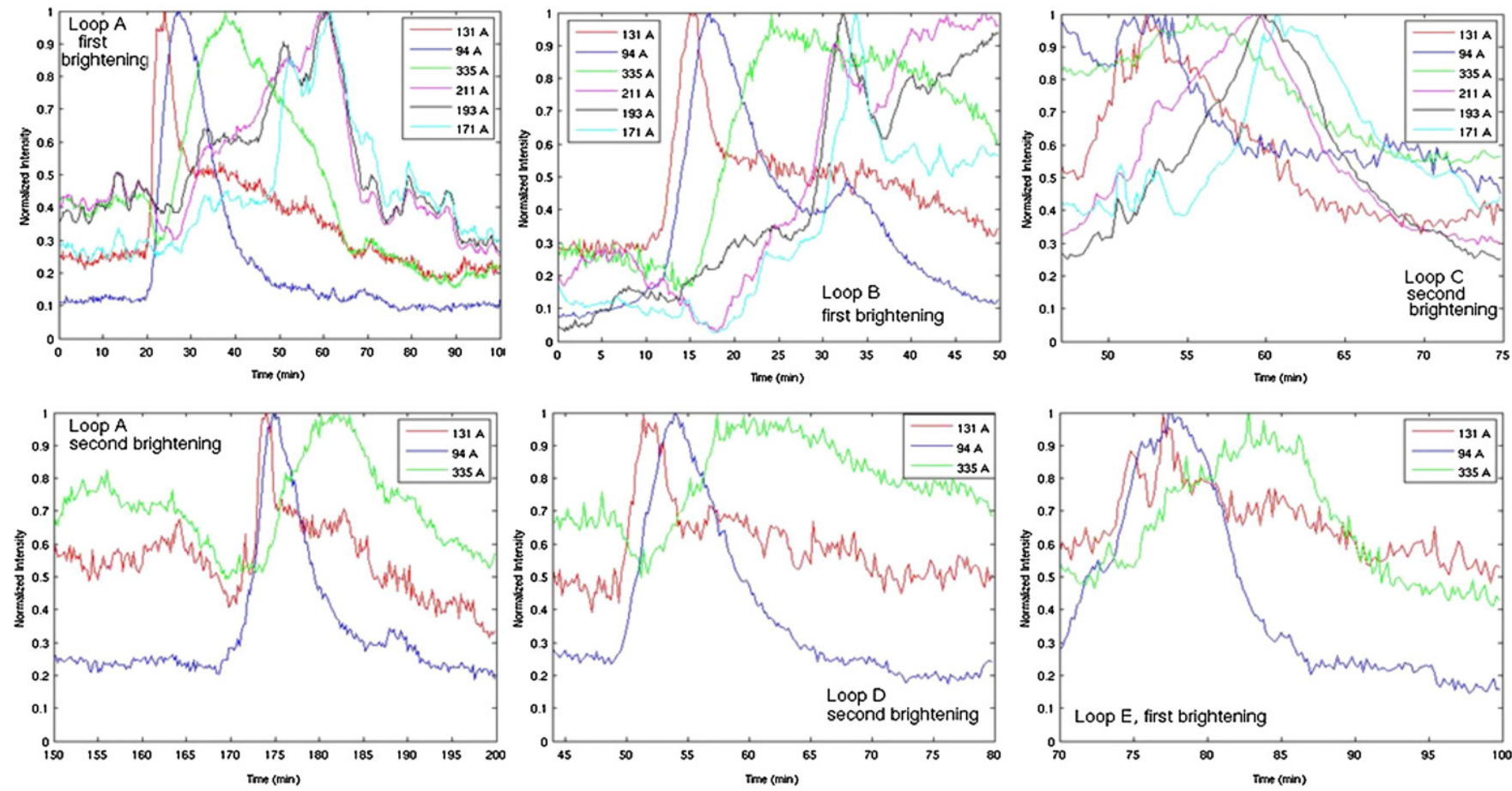

Figure 6. Intensity time traces around particular brightenings. Top row: possible nanoflare storms with progressive cooling in the six EUV channels. Bottom row: partial "cascade" with progressive cooling in the three hot channels.

(A color version of this figure is available in the online journal.)

by the hot $94 \AA$ signal and the cooler channels in progressive order (hot 131, hot 94, 335, 211, 193, 171). For the first brightening in Loop A the peak in the hot 131 signal occurs at 23.8 minutes, and the rest at time delays of $(3.2,14.0,36.8$, $37.0,37.8)$ minutes. In the case of the first brightening in Loop
B the hot 131 peak occurs at 15.6 minutes and the rest at time delays of $(1.6,8.6,15.6,16.8,18.2)$ minutes, and for the second brightening in Loop $C$ the hot 131 peak occurs at 52.4 minutes and the rest at time delays of $(1.4,3.4,7.4,7.4,8.4)$ minutes. While there is no common value for the time difference between 
the peak times in the three examples, in all cases the $131 \AA$ and $94 \AA$ peaks are close in time. Also the $211 \AA$, $193 \AA$, and $171 \AA$ are grouped together. The $335 \AA$ signal has a wide peak and is more separated in time from the hot channels and the warm/cool channels.

The nanoflare storm model assumes that the strands comprising a loop are impulsively heated at different, independent times and with independent cooling times (Cargill 1994; Mulu-Moore et al. 2011). The net effect leads to a thermal distribution for the loop. Viall \& Klimchuk (2011) have used the EBTEL (Klimchuk et al. 2008) model to simulate nanoflare storms of varying duration and intensity. One of their cases, consisting of high energy individual nanoflares with a triangular shape of $500 \mathrm{~s}$ duration, leads to intensity time series with the same salient characteristics as those obtained in the present examples (Figure 3(b) in Viall \& Klimchuk 2011). When another nanoflare storm occurs and there has not been enough time for the energy to dissipate through the cooler channels, the brightenings present partial "cascades" only in the hotter three channels. In Figure 6 the second brightening in Loop A, the second brightening in Loop $\mathrm{D}$, and the first brightening in Loop E present peaks for the 131, 94, $335 \AA$ signals at $(174$, $174.8,182)$ minutes, $(51.6,54.2,57.6)$ minutes, and $(77,77.6$, $82.8)$ minutes, respectively.

\section{LOOP HEATING PROCESS}

The results in the previous section indicate the presence of an impulsive heating mechanism. The presence of very hot plasmas is a characteristic of nanoflare heating. To gain more insight into the process here we calculate the time evolution of the electron temperature and emission measure for two characteristic heating events.

For this purpose we have adapted the method developed by Aschwanden \& Boerner (2011) and Aschwanden et al. (2013). The flux in the six coronal wavelength filters can be represented by

$$
\begin{aligned}
F_{\lambda}(t, x) & =\int \frac{d \operatorname{EM}(T, t, x)}{d T} R_{\lambda}(T) d T \\
& =\sum_{\mathrm{k}} \operatorname{DEM}\left(T_{k}, t, x\right) R_{\lambda}\left(T_{k}\right) \Delta T_{k}
\end{aligned}
$$

where the $F_{\lambda}(t, x)$ are the background-subtracted intensities along the loop coordinate $x$ at time $t$, and the $R_{\lambda}(T)$ are the instrumental response functions. We approximated the differential emission measure $d \operatorname{EM}(T, t, x) / d T(\mathrm{DEM})$ via a superposition of Gaussian functions

$$
\begin{aligned}
\frac{d \operatorname{EM}(T, t, x)}{d T}= & \sum_{p=1}^{n} \operatorname{EM}_{p}(t, x) \\
& \times \exp \left(-\frac{\left[\log (T)-\log \left(T_{p}(t, x)\right)\right]^{2}}{2 \sigma_{p}^{2}(t, x)}\right) .
\end{aligned}
$$

A calculation at each space time point of the peak emission measures $\operatorname{EM}_{p}(t, x)$, temperatures $T_{p}(t, x)$, and widths $\sigma_{p}(t, x)$, was done via chi-square optimization of a single Gaussian model and a double Gaussian model with equal amplitudes. The latter is expected to provide a more accurate analysis of the data (Reale et al. 2009; Sylwester et al. 2010), and it is particularly relevant in our case since the AR core loops, which are bright in the $94 \AA$, have the potential of being multithermal (Aschwanden et al. 2013). Because there are only six data points for each fit we have found it necessary to limit the fit to five parameters and we therefore specify equal amplitudes. We used the latest version, V4 (2014 March), of the AIA response functions obtained from SSWIDL. In the single Gaussian model the optimization was performed via a look-up table leading to an estimate of the peak quantities. For the double Gaussian model we used the Matlab routine FitChiSquare, which implements a generalized nonlinear optimization according to the algorithm developed by Press et al. (1986). In contrast to the calculations in the previous references in which a fit is performed over the width of the loop and over the six intensity measurements, here we only have available the six intensities to perform the fit. Testa et al. (2012) applied the Monte Carlo Markov chain forwarding method to investigate the temperature diagnostics of synthetic AIA data and found inaccuracies due to the small number of constraints provided by the AIA data and the broad response functions. Therefore the quantitative results have to be taken with caution but, as will be shown, the qualitative joint behavior of the temperature and electron density provides useful information on the properties of the heating process.

We define the emission-weighted temperature (Chitta et al. 2013) as:

$$
T e(t, x)=\frac{\sum_{k} \operatorname{DEM}\left(T_{k}, t, x\right) T_{k} \Delta T_{k}}{\sum_{k} \operatorname{DEM}\left(T_{k}, t, x\right) \Delta T_{k}}
$$

where DEM is the differential emission measure introduced in Equation (2), $T_{k}$ is the temperature, and $\Delta T_{k}=0.05$ actually refers to intervals in the value of $\log (T)$. The sum in $\log (T)$ ranges between 5.35 and 7.35. The electron density is estimated by calculating $n e=(\mathrm{EM} / w)^{1 / 2}$ where $\mathrm{EM}$ is the emission measure obtained by integrating $\operatorname{DEM}(T)$ over the temperature $T$ and $w$ is the loop width, which has been taken to be constant at 9 pixels or 3.9 Mm (Aschwanden et al. 2013).

To aid physical interpretation of the evolution of the loop temperatures and electron densities we focus on loops $\mathrm{A}$ and B. These each present an early main brightening with a narrow localization in time and so permit an uncluttered interpretation of the aftermath. Given the limited number of data points the $\chi^{2}$ goodness of fit values for the double Gaussian model are larger than for the single Gaussian model but there is generally good agreement between the results obtained with the two models. For Loop A using the single Gaussian approach $86 \%$ of the pixels had $\chi^{2}<4$, whereas for the double Gaussian model only $53 \%$ of the pixels had $\chi^{2}<4$. For Loop B the results were: single Gaussian $85 \%$ and double Gaussian $47 \%$. Upon closer inspection, however, the double Gaussian method better represents physical details that are not captured by the single Gaussian model, such as the temperature increase associated with the small second brightening in Loop A (Figure 5). Thus henceforth we will take the double Gaussian approach.

The emission-weighted temperature Te of Loop A obtained with the double Gaussian model reached a maximum temperature of 6.5 MK, and the maximum density occurs at the "peak time" (Aschwanden \& Shimizu 2013) with maximum value of $n_{p}=9.5 \times 10^{9} \mathrm{~cm}^{-3}$ and a corresponding peak temperature $T_{p}=3.8 \mathrm{MK}$. A similar analysis for Loop B gives a maximum temperature of $11 \mathrm{MK}$, and the maximum electron density $n_{p}=8.3 \times 10^{9} \mathrm{~cm}^{-3}$ and corresponding peak temperature $T_{p}=6.2 \mathrm{MK}$. We note that since a $100 \%$ filling factor has been assumed across the loop and an offset has been subtracted the maximum density values are lower limits. 

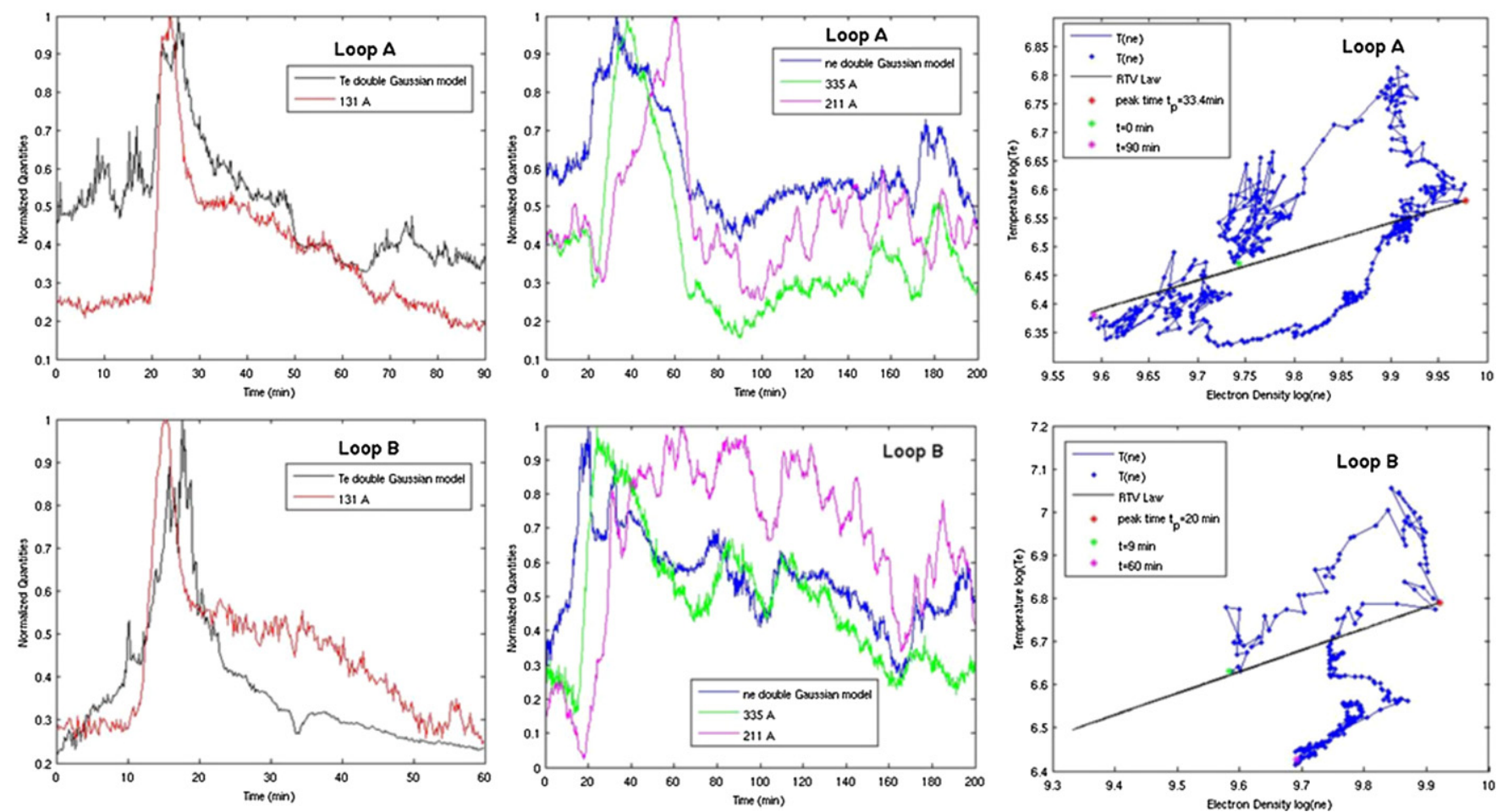

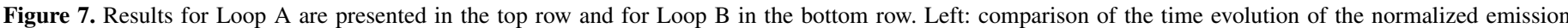

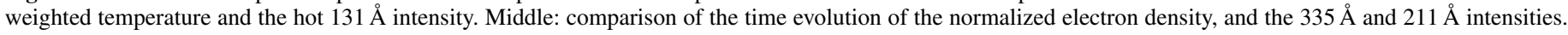

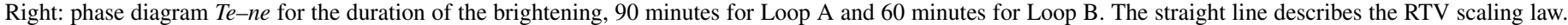
The points corresponding to the peak time for the maximum density as well as the beginning and end times are given for reference.

(A color version of this figure is available in the online journal.)

In Figure 7 (top right) we plot the $T e-n e$ phase diagram for the first brightening $(0<t<90$ minutes $)$ of Loop A. We superimpose the line corresponding to the Rosner-Tucker-Vaiana (1978) scaling law (RTV) $T e \propto n e^{1 / 2}$ with the constant of proportionality fixed by assuming that the RTV relation is satisfied when $n e$ and $T e$ have their peak values $\left(n_{p}, T_{p}\right)$. Also indicated in the figure are the points corresponding to the peak time as well as the beginning and end times of the brightening. The system phase proceeds in time in a clockwise sense. Concentrating on the times around the brightening ( 9 minutes $<t<$ 60 minutes) the $T e-n e$ phase diagram shows the tendency for the data points to be above the RTV line during the heating phase and below for the cooling phase. This is precisely the behavior encountered in a hydrodynamic simulation for an impulsively heated single loop (Figure 4, Aschwanden \& Shimizu 2013). Figure 7 (bottom right) shows the same behavior for Loop B. In earlier work Jakimiec et al. (1999) had investigated the density-temperature relation in a one-dimensional hydrodynamic model with a sudden energy release, which corresponds to the schematic description in Reale $(2007,2010)$. We differ from these analyses in that the present results have a less steep rise in temperature.

For steady loops it would be possible to apply the RTV scaling laws to obtain the heating rate as a function of the loop length and maximum temperature. Since the loops under study are not steady we must look for a proxy for the energy input by comparing the time evolution of the intensity signals with those of the emission-weighted temperature and the electron density. For this purpose we consider these quantities normalized at their maximum over the 270 minute observation runs. Figure 7 (topleft) compares the normalized $T e$ time series and the normalized $131 \AA$ intensity for the first brightening in Loop A. Figure 7 (top-middle) compares the corresponding normalized ne time series, and the normalized $335 \AA$ and $211 \AA$ intensities. The $131 \AA$ signal has a peak at 23.8 minute that is followed by the temperature peak 1.8 minutes later at 25.6 minutes and the maximum density 9.6 minutes later at 33.4 minutes. For Loop B (Figure 7, bottom row) the progression of maxima is $131 \AA$ : $T e: n e$ at 15.6:17.6:27.6 minutes. While the temperature drops to its initial value during the duration of the brightening, the density increases and is controlled by the presence of the cooler plasma filling the loop as indicated by the temporal evolution of the $335 \AA$ and 211 A signals. Qualitatively, it is clear that impulsive heating is taking place with a fast increase and decrease in the temperature followed by a more gradual process in the density. The temperature peaks closely follow the hot $131 \AA$ intensity maxima. This delay probably arises from inaccuracies in the inversion method, but the result nevertheless supports the notion that the hot $131 \AA$ signal is directly linked to the energy deposition and can serve as a proxy measure for the same.

At this point, we can further estimate how the energy released in the loops compares to that of the flares classified in Aschwanden \& Shimizu (2013). We have applied their formula for the thermal energy:

$$
E_{\mathrm{th}}(t)=3 n e(t) k_{B} T e(t) V(t)
$$

where $k_{B}$ is the Boltzmann constant and $V$ is the flare volume. We have approximated the loop volumes by a cylinder over the inter-moss region. This leads to a peak thermal energy of $\sim 10^{27.5}$ erg. A similar calculation for the $\mathrm{C} 1.2$ class flare studied by Petkaki et al. (2012) and using their published values gives a thermal energy of $\sim 10^{28.5} \mathrm{erg}$. This order of magnitude difference between the thermal energies associated with the loop 
brightenings versus the $\mathrm{C} 1.2$ class flare is compatible with the ratios in peak intensities described in Section 2. The thermal energy of the $\mathrm{C}$ class flare is about one to two orders of magnitude smaller than the values for the $\mathbf{M}$ class flares encountered by Aschwanden \& Shimizu.

\section{POWER LAW SPECTRA OF INTENSITY FLUCTUATIONS}

In the preceding analyses we have encountered strong evidence that the class of nonsteady loops in our study are impulsively heated in a manner compatible with nanoflare storms. In a classic paper Hudson (1991) determined that the energy distribution of nanoflare type events also obeys a power law as previously encountered for flares. In general these phenomena are characterized by probability density functions with power law form (Aschwanden 2004 presents a summary of scaling exponents). Since we are dealing with nonsteady processes we cannot directly relate observed intensity changes to inputs of dissipated energy (Martens 2010).

However, we find evidence that the hot $131 \AA$ intensity signal can be useful as a proxy for the energy input. For one thing, abrupt loop intensity brightenings commonly begin with a sharp impulse in the hot $131 \AA$ intensity followed in sequence by progressively broader, smoother, and later increases in the hot $94 \AA$ intensity and then the $335 \AA$ intensity. The hot $131 \AA$ intensity is the one least affected by processes within the loop, like cooling or plasma flows, and it is the best available indicator for energy input from the outside. To perform a statistically significant study of the probability density function of intensity event strengths we would need a much larger sample of loops. Acquisition of a significantly greater data base is a goal for future research. For now we consider power spectra of the various intensity time series. To compute the average power spectrum we first calculate the spectrum of background-subtracted intensity time series in a given wavelength band for each of the five loops, and then average the five spectra at each frequency. The results are then presented in log-log plots. Figure 8 (upper left) shows the average power spectrum for the hot $131 \AA$ intensity as well as for the total $131 \AA$ intensity signals. The spectrum for the hot 131 signal has a $1 / f^{\beta}$ form with two regimes. These show a scaling exponent $\beta=1.31 \pm 0.07$ for $f<0.1 \mathrm{~min}^{-1}=1.67 \times 10^{-3} \mathrm{~Hz}$, and $\beta=2.71 \pm 0.16$ for $0.1<f<0.7 \mathrm{~min}^{-1}$ or $1.67 \times 10^{-3}$ $<f<1.17 \times 10^{-2} \mathrm{~Hz}$. For higher frequencies we find white noise. In comparison, for $f<0.1 \mathrm{~min}^{-1}$, the spectrum for the total 131 signal has a scaling exponent of $\beta=1.81 \pm 0.12$. For this low frequency regime the difference between the slopes of $0.50 \pm 0.14$, corresponding to $3.6 \sigma$, is significant. These results indicate that while the "hot" signal has the properties of " $1 / f$ noise" characterized by a scaling exponent $0.5<\beta<1.5$, the "total" signal includes contributions from a different process.

As is well known, $1 / f$ noise is encountered in many examples in nature. In particular Ueno et al. (1997) interpreted the power spectra for solar X-ray fluctuations measured by the GOES 6 satellite in terms of a superposition of flare like impulses with exponential relaxation functions $\sim \exp (-t / \tau)$ and a wide distribution of decay times $\sim(10<\tau<140$ minutes $)$. If the impulse response function obeys a power law $\sim t^{-\gamma}$ then the scaling exponent is given by $\beta=2(1-\gamma)$ (Lowen \& Teich 1990). In the present case of our hot $131 \AA$ spectra this would imply a power law index $\gamma=0.36$ applied to the range $f<0.1 \mathrm{~min}^{-1}=6 \mathrm{hr}^{-1}$. This includes the minimum frequency of 2-3 heating events per hour encountered by UW14. Moreover, the upper limit of the scaling range is compatible with their maximum count of $\sim 5$ events per hour (Figure 6 , left, in their paper). Although their nanoflare storm simulation used square impulses it is interesting to consider the possibility of a variation with pulses with power law decays. As reported in their paper, UW14 have run simulations with $\sim 9$ events per hour, obtaining an intensity envelope which is compatible with the observations. This has led them to conjecture that the true event frequencies could be much higher. In the present case we find that the power law spectrum has a break at $f \approx 0.1 \mathrm{~min}^{-1}$, and the scaling exponent changes to $\beta=2.71$ with Hurst exponent $H=(\beta-1) / 2=0.86$, implying the higher frequency time series has a long-term positive autocorrelation (Schroeder 1991, chap. 5).

To interpret the power spectrum with three scaling regimes we have compared our spectra to the spectra of intermittent energy dissipation derived from a numerical model in which the magnetic footpoint motions in the photosphere lead to the injection of magnetohydrodynamic (MHD) turbulence into coronal loops, where the latter is stored and dissipated (Nigro et al. 2004; Reale et al. 2005). This "hybrid" shell model, based on reduced MHD (RMHD), was used to numerically model the nonlinear couplings between magnetic and velocity fluctuations that transfer energy from large to small scales where dissipation then converts the turbulent energy to heat. The coronal nanoflares can be explained as the intermittent energy releases due to dissipation in the MHD turbulence process. The simulation does not describe the physical mechanism of magnetic reconnection, because it uses a shell model, which does not allow one to keep the details of the magnetic topology in the directions perpendicular to the main magnetic field $\mathbf{B}_{0}$. However it provides the capability to reach very large Reynolds numbers. In particular, the model is derived starting from the RMHD equations and performing the Fourier decomposition of the field components perpendicular to $\mathbf{B}_{0}$, while the dependence on the space variable $x$ along $\mathbf{B}_{0}$ is kept. This $\mathbf{k}_{\perp}$-space includes only concentric shells of wave-vectors with exponentially growing radius $k_{n}=k_{0} 2^{n}\left(k_{0}=2 \pi / L_{\perp}\right.$, where $L_{\perp}$ is the width of the loop cross section). In each shell, two scalar complex amplitudes, $u_{n}(x, t)$ and $b_{n}(x, t)$, are considered for the perpendicular velocity and magnetic field, respectively. It is imposed that the nonlinear coupling of the field amplitudes among shells conserve quadratic invariants: total energy, cross helicity, and squared magnetic potential. The evolution equations for dynamical variables $u_{n}(x, t)$ and $b_{n}(x, t)$ are hence derived in terms of the Alfvén speed (see Nigro et al. 2004 for details):

$$
\begin{gathered}
\frac{\partial u_{n}(x, t)}{\partial t}-\frac{\partial b_{n}(x, t)}{\partial x}=-v k_{n}^{2} u_{n}(x, t)+i k_{n} \\
\quad \times\left(u_{n+1} u_{u+2}-b_{n+1} b_{n+2}-\frac{5}{8}\left(u_{n-1} u_{n+1}\right.\right. \\
\left.\left.-b_{n-1} b_{n+1}\right)+\frac{1}{16}\left(u_{n-2} u_{n-1}-b_{n-2} b_{n-1}\right)\right)^{*} \\
\frac{\partial b_{n}(x, t)}{\partial t}-\frac{\partial u_{n}(x, t)}{\partial x}=-\mu k_{n}^{2} b_{n}(x, t)+i k_{n} \\
\quad \times\left(\frac{1}{12}\left(u_{n+1} b_{n+2}-b_{n+1} u_{n+2}\right)+\frac{1}{6}\left(u_{n-1} b_{n+1}\right.\right. \\
\left.\left.-b_{n-1} u_{n+1}\right)+\frac{1}{3}\left(u_{n-2} b_{n-1}-b_{n-2} u_{n-1}\right)\right)^{*}
\end{gathered}
$$

with $n=0,1, \ldots, n_{\max }\left(n_{\max }=11\right)$. Here $v$ is the dimensionless viscosity, and $\mu$ is the dimensionless magnetic diffusivity. 

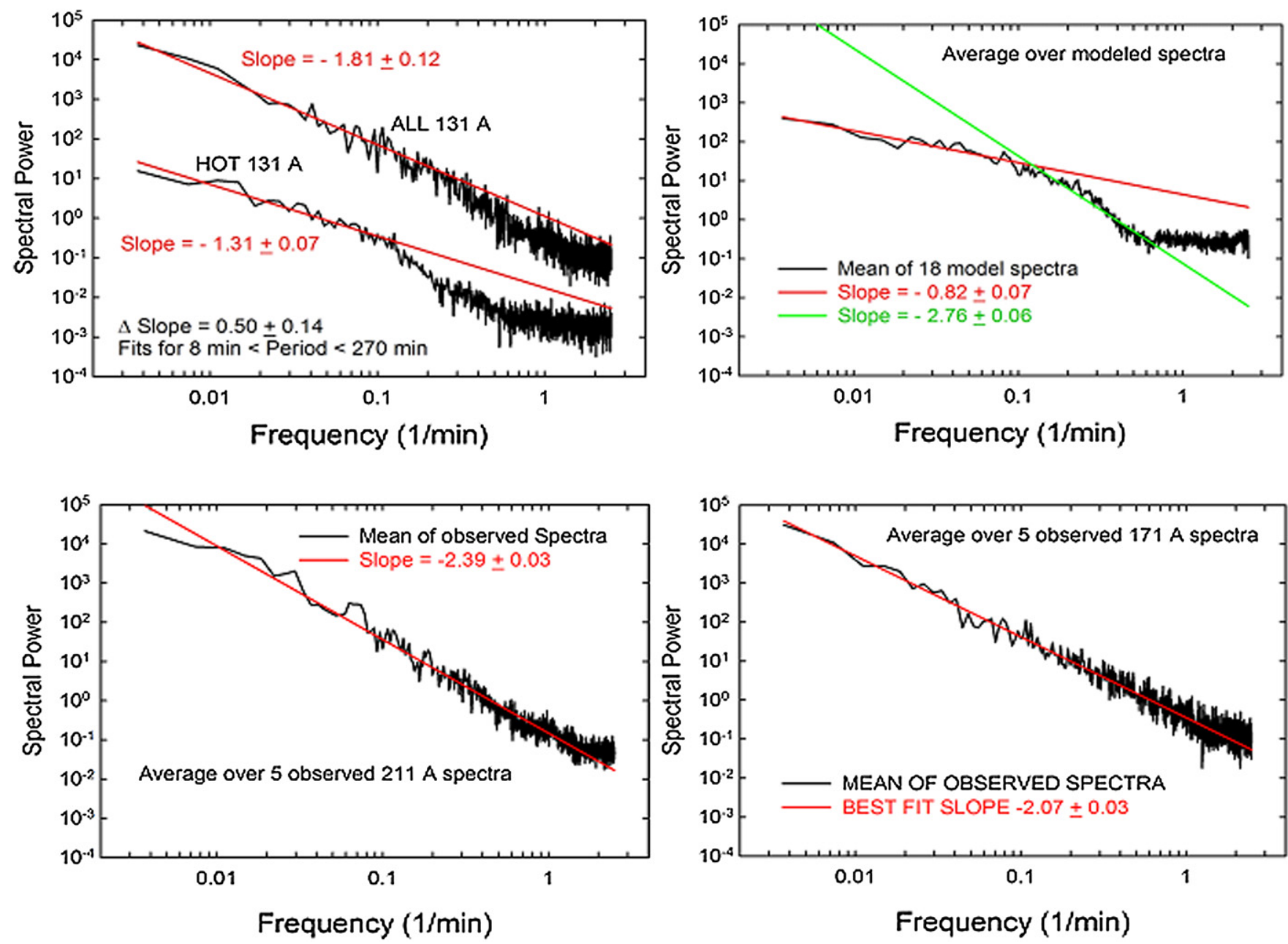

Figure 8. Average power spectra for different time series. Top-left: hot 131 and total 131 intensities for Loops A-E. Top-right: energy dissipation spectra from MHD turbulence model. Bottom-left: spectra of 211 A intensities for Loops A-E. Bottom-right: 171 Å intensities for Loops A-E.

(A color version of this figure is available in the online journal.)

The forcing for the velocity is applied to the first three shells $(n=0,1,2)$ in order to reproduce the photospheric motions concentrated at larger spatial scales $\sim L_{\perp}$. The forcing is realized by random Gaussian-distributed signals with an amplitude of $\sim 1 \mathrm{~km} \mathrm{~s}^{-1}$ and a correlation time of $\sim 5$ minutes. This is typical of photospheric motions. Total reflection of the field amplitudes is imposed at both boundaries, that is, at the two footpoints. Therefore Equations (5) and (6) describe the evolution of velocity and magnetic field fluctuations $u_{n}$ and $b_{n}$ propagating along the loop, i.e., along $x$, at the Alfvén speed. On the RHS of Equations (5) and (6) the nonlinear coupling terms describe the turbulent cascade of the energy that is injected at larger scales, toward the smaller dissipative scales. The model equations were numerically solved using values for the dimensionless parameters corresponding to a typical coronal loop. Therefore our numerical results reproduce the evolution of the plasma in a typical loop characterized by a longitudinal length $L=3 \times 10^{4}$ $\mathrm{km}$, an aspect ratio $R=L / L_{\perp}=30 / 2 \pi$, Alfvén velocity $c_{A}=$ $2 \times 10^{3} \mathrm{~km} \mathrm{~s}^{-1}$, and mass density $\rho=1.67 \times 10^{-16} \mathrm{~g} \mathrm{~cm}^{-3}$. Very small dimensionless dissipation coefficients $v=\mu=10^{-7}$ were used.

Figure 8 (top right) shows the average over the spectra of 18 segments of the modeled energy dissipation data each with a 270 minute length and $12 \mathrm{~s}$ cadence to match our observational data. The average spectrum has three scaling regimes with break points comparable to those of the hot $131 \AA$ intensity spectrum and scaling exponents $(0.82,2.76,0)$. While the precise values differ from those of the data, they are not far apart, and the qualitative properties are the same indicating a $1 / f$ process for the lower frequencies, strong persistence with Hurst exponent $H=0.88$ for the intermediate regime, and white noise at higher frequencies. The notable resemblance of the model spectra to the hot $131 \AA$ spectra further strengthens the idea that the hot $131 \AA$ signal can serve as a proxy for the dissipated energy input to the loop. In the calculations leading to Figure 8, the assumed width of the loops studied was nine pixels or $3.6 \mathrm{Mm}$ (see Figure 1). Thus, if the loops were to undergo transverse movements of $\sim 2 \mathrm{Mm}$ or more over the 270 minute observational time span, then spurious intensity fluctuations might appear. Because of its importance to our results, we have checked the robustness of the hot $131 \AA$ mean spectrum in Figure 8 (top left) against transverse motions of the loops by means of increasing the assumed loop width and looking for changes in the spectrum. We have recalculated the hot $131 \AA$ spectra with assumed loop widths of 7.2 Mm and 14.4 Mm. We find that the three-scalingregime pattern is preserved in all cases. The scaling exponents for the low-frequency $1 / f$ portion of the spectra change from $1.31 \pm 0.07$ for loop width $3.6 \mathrm{Mm}$ to $1.32 \pm 0.09$ for loop 
width $7.2 \mathrm{Mm}$ and to $1.40 \pm 0.09$ for loop width $14.4 \mathrm{Mm}$. These are all consistent within estimated error limits.

Finally we have considered the effect of using different calibration factors when extracting the hot component of the $131 \AA$ signal. With a coefficient $a=0.032$ we find that the long period exponent of the average spectrum is $-1.23 \pm 0.08$. Comparing to the case presented in Figure 8 with $a=0.044$ and scaling exponent $-1.31 \pm 0.07$ shows that these values are consistent within their error bars.

We have also investigated the intensity power spectra for the other EUV channels. No scaling ranges were found for the hot $94 \AA$ and the $335 \AA$ signals. Figure 8 (bottom) shows the results for the $211 \AA$ and $171 \AA$ signals. The scaling range in these cases and for the $193 \AA$ signal (not shown) approximately extends over all the available frequencies: from the Nyquist frequency $2.5 \mathrm{~min}^{-1}$ to $0.004 \mathrm{~min}^{-1}$. The scaling exponents for the $171 \AA, 193 \AA$, and $211 \AA$ power spectra are $\beta=2.07 \pm 0.03$, $2.30 \pm 0.03$, and $2.39 \pm 0.03$, respectively. These correspond to Hurst exponents $0.54,0.65$, and 0.70 , respectively, indicating the presence of long-term persistence in these signals. When the assumed loop width is increased to $14.4 \mathrm{Mm}$, the scaling exponents become $1.98 \pm 0.02,2.10 \pm 0.02$, and $2.12 \pm 0.02$. So the intensity variations become more nearly Brownian in nature. The energy released into the loops in these warm channels follows a different process than the energy originally deposited (using the hot $131 \AA$ signal as a proxy). We propose that the $94 \AA$ fluctuations are related to the hot $131 \AA$ input proxy by cooling processes from $10 \mathrm{MK}$ to $7 \mathrm{MK}$. The $171 \AA$, $193 \AA$, and $211 \AA$ fluctuations appear to behave quite differently, and we propose that they are governed by the later evaporation of chromospheric ions into the loops where they radiate persistently. The $335 \AA$ emission pattern appears to include both processes.

\section{SUMMARY OF RESULTS AND DISCUSSION}

The detailed time evolution of loops A-E, for what we have identified as the inter-moss region based on the properties of the photospheric LOS magnetic field, shows a pattern of brightenings in which the hot $131 \AA$ intensity peak was followed quickly by the hot $94 \AA$ signal and then the cooler channels in progressive order of cooling, resembling the simulation of a high intensity nanoflare storm (Viall \& Klimchuk 2011). The same result has been encountered by Petkaki et al. (2012) in the case of an isolated C1-class solar flare. In these cases we found that the temperature and electron density are functionally related in a manner compatible with an impulsive heating process and with the hot $131 \AA$ signal preceding the temperature peak. The density increases as the loops fill with plasma which radiates in the cooler channels. When the heating impulses are closer together in time we encounter also examples of higher frequency heating with partial cascades only in the three hottest channels. Since the hot $131 \AA$ signal has a clear association with the temperature increase we can use it as a proxy for the energy deposition. We find that the average power spectra in the hot $131 \AA$ signals presents a $1 / f^{\beta}$ form with three scaling regimes. For $f<0.1 \mathrm{~min}^{-1}$ we find the scaling exponent $0.5<\beta<1.5$ which corresponds to $1 / f$ noise. A possible mechanism to explain this type of scaling is through a model characterized by the superposition of impulses with response functions obeying a power law (Lowen \& Teich 1990). This is reminiscent of the prescription for the nanoflare storm models with the difference that those use simplified triangular or square pulses. The range of scaling frequencies is compatible with the statistical analysis of UW14 who find a characteristic rate of 2-3 heating events per hour in hot $94 \AA$ signals in active regions. Their simulations suggest that the actual rate could be much higher. However our results indicate that for $f>0.1 \mathrm{~min}^{-1}$ the scaling exponent $\beta$ $\geqslant 2$ indicating the presence of long term persistence. It would be very useful to calculate the power spectra of nanoflare models with different impulsive event rates.

We have found that the hybrid shell model for loop heating via the dissipation of turbulent energy presents spectra with the same scaling properties and comparable break points as the hot $131 \AA$ data. In this context, the impulsive events result from the intermittent turbulent energy deposition. We note that although this model does not describe some spatial details like the precise mechanism of magnetic reconnection, it is able to reach very large Reynolds numbers, not yet accessible in the direct numerical simulations of 3D MHD, and therefore it is very powerful in the reproduction of a very long dissipative signal (with a wide spectrum), rich in the number of intense energy releases. This has proven to be crucial for a comparison with the data. On the other hand models for 3D MHD turbulence have shown that the kind of fast magnetic reconnection that is required for flare and flare-like processes can be achieved as a consequence of the wandering of the stochastic magnetic field at sub-resolution scales (Lazarian \& Vishniac 1999; Eyink et al. 2011; Eyink et al. 2013; Browning \& Lazarian 2013). The fact that the turbulent model with its scaling properties fits the observations suggests the presence of fast heating mechanisms.

AIA data are courtesy of NASA/SDO and the AIA science team. A.C.C. acknowledges support from the Interdisciplinary Research Institute for the Sciences (IRIS) at California State University Northridge. We thank P. Testa and the anonymous referee for helpful suggestions.

\section{REFERENCES}

Antiochos, S. K., Karpen, J. T., DeLuca, E. E., Golub, L., \& Hamilton, P. 2003, ApJ, 590, 547

Aschwanden, M. J. 2004, Physics of the Solar Corona: An Introduction (New York: Springer)

Aschwanden, M. J., \& Boerner, P. 2011, ApJ, 732, 81

Aschwanden, M. J., Boerner, P., Schrijver, C. J., \& Malnushenko, A. 2013, SoPh, 283, 5

Aschwanden, M. J., \& Nightingale, R. W. 2005, ApJ, 633, 499

Aschwanden, M. J., \& Shimizu, T. 2013, ApJ, 776, 132

Asgari-Targhi, M., \& van Ballegooijen, A. A. 2012, ApJ, 746, 81

Asgari-Targhi, M., van Ballegooijen, A. A., Cranmer, S. R., \& DeLuca, E. E. 2013, ApJ, 773, 111

Boerner, P. F, Testa, P., Warren, H., Weber, M. A., \& Schrijver, C. J. 2014, SoPh, 289, 2377

Bradshaw, S. J. 2008, A\&A, 486, L5

Browning, P., \& Lazarian, A. 2013, SSRv, 178, 325

Cargill, P. J. 1994, ApJ, 422, 381

Cargill, P. J., \& Klimchuk, J. A. 1997, ApJ, 478, 799

Chitta, L. P., Kariyappa, R., van Ballegoijen, A. A., et al. 2013, ApJ, 768, 32

Del Zanna, G. 2013, A\&A, 558, 73

Del Zanna, G., \& Mason, H. E. 2003, A\&A, 406, 1089

Eyink, G., Vishniac, E., Lalescu, L., et al. 2013, Natur, 497, 468

Eyink, G. L., Lazarian, A., \& Vishniac, E. T. 2011, ApJ, 743, 51

Hudson, H. 1991, SoPh, 133, 357

Jakimiec, J., Sylwester, B., Sylwester, J., et al. 1992, A\&A, 253, 269

Klimchuk, J. A. 2000, SoPh, 193, 53

Klimchuk, J. A. 2006, SoPh, 234, 41

Klimchuk, J. A. 2009, SoPh, 234, 41

Klimchuk, J. A., Patsourakos, S., \& Cargill, P. J. 2008, ApJ, 682, 1351

Lazarian, A., \& Vishniac, E. T. 1999, ApJ, 517, 700

Lemen, J. R., Title, A. M., Akin, D. J., et al. 2012, SoPh, 275, 17

Lowen, S. B., \& Teich, M. C. 1990, IEEE Trans. Inf. Theory, 36, 1302

Martens, P. 2010, ApJ, 714, 1290

Mulu-Moore, F. M., Winerbarger, A. R., \& Warren, H. P. 2011, ApJL, 742, L6 
Mulu-Moore, F. M., Winebarger, A., Warren, H. P., \& Aschwanden, M. 2011, ApJ, 733, 59

Nigro, G., Malara, F., Carbone, V., \& Veltri, P. 2004, PhRvL, 92, 194501

Parker, E. N. 1988, ApJ, 330, 474

Patsourakos, S., \& Klimchuk, J. A. 2009, ApJ, 696, 760

Pesnell, W. D., Thompson, B. T., \& Chamberlin, P. C. 2012, SoPh, 275, 3

Petkaki, P., Del Zanna, G., Mason, H. E., \& Bradshaw, S. J. 2012, A\&A, 547, 2

Petralia, A., Reale, F., Testa, P., \& Del Zanna, G. 2014, A\&A, 564, 3

Press, W. H., Teukolsky, S. A., Vetterling, W. T., \& Flannery, B. P. 1986, Numerical Recipes, the Art of Scientific Computing (Cambridge: Cambridge Univ. Press)

Reale, F. 2007, A\&A, 471, 271

Reale, F. 2010, LRSP, 7, 5

Reale, F., Guarrasi, M., Testa, P., et al. 2011, ApJL, 736, L16

Reale, F., McTiernan, J. M., \& Testa, P. 2009, ApJ, 704, 58

Reale, F., Nigro, G., Malara, F., Peres, G., \& Veltri, P. 2005, ApJ, 633, 489

Reale, F., \& Orlando, S. 2008, ApJ, 684, 715

Reale, F., Testa, P., Klimchuk, J. A., \& Parenti, S. 2009, ApJ, 698, 756

Schroeder, M. 1991, Fractals, Chaos, Power Laws (New York: W. H. Freeman \& Company)

Rosner, R., Tucker, W. H., \& Vaiana, G. S. 1978, ApJ, 220, 643

Sylwester, B., Sylwester, J., \& Phillips, K. J. H. 2010, A\&A, 514, 82

Terzo, S., \& Reale, F. 2010, A\&A, 515, 7
Testa, P., De Pontieu, B., Martínez-Sykora, J., Hansteen, V., \& Carlsson, M. 2012, ApJ, 758, 54

Testa, P., De Pontieu, B., Martínez-Sykora, J., et al. 2013, ApJL, 770, L1

Testa, P., \& Reale, F. 2012, ApJL, 750, L10

Testa, P., Reale, F., Landi, E., DeLuca, E., \& Kashyap, V. 2011, ApJ, 728, 30

Tripathi, D., Klimchuk, J. A., \& Mason, H. E. 2011, ApJ, 740, 111

Tripathi, D., Mason, H. E., Dwivendi, B. N., delZanna, G., \& Young, P. R. 2009, ApJ, 694, 1256

Ueno, S., Mineshige, S., Negoro, H., Shibata, K., \& Hudson, H. 1997, ApJ, 484,920

Ugarte-Urra, I., \& Warren, H. P. 2012, ApJ, 761, 21

Ugarte-Urra, I., \& Warren, H. P. 2014, ApJ, 783, 12

Ugarte-Urra, I., Warren, H. P., \& Brooks, D. H. 2009, ApJ, 695, 642

Van Ballegooijen, A. A., Asgari-Targui, M., Cranmer, S. R., \& DeLuca, E. E. 2011, ApJ, 736, 3

Veltri, P., Nigro, G., Malara, F., Carbone, V., \& Mangeney, A. 2005, Nonlinear Proc. Geophys., 12, 245

Viall, N. M., \& Klimchuk, J. A. 2011, ApJ, 738, 24

Viall, N. M., \& Klimchuk, J. A. 2012, ApJ, 753, 35

Warren, H. P., Brooks, D. H., \& Winebarger, A. R. 2011, ApJ, 734, 90

Warren, H. P., Winebarger, A. R., \& Brooks, D. H. 2012, ApJ, 759, 14

Winebarger, A. R., Schmelz, J. T., Warren, H. P., Saar, S. H., \& Kashyap, V. L. 2011, ApJ, 740, 2 